ESAIM: PROCEEDINGS, April 2009, Vol. 26, p. 55-64

H. Ammari, Editor

\title{
GENERALIZED FOURIER TRANSFORMS, INVERSE PROBLEMS, AND INTEGRABILITY IN $4+2$
}

\begin{abstract}
A.S. FOKAS ${ }^{1}$
Abstract. Three different types of generalized Fourier transforms are discussed, which appear in the following: (a) The medical imaging technique called Single Photon Emission Computerized Tomography (SPECT). (b) The solution of the Cauchy problem of integrable nonlinear evolution PDEs in $4+2$, i.e. in four spatial and two temporal dimensions. (c) The characterization of the generalized Dirichlet to Neumann map for integrable nonlinear evolution PDEs in $1+1$, i.e. in one spatial and one temporal dimensions.
\end{abstract}

\section{INTRODUCTION}

Let the scalar valued function $\mu(x, y, k)$ satisfy the eigenvalue equation $L \mu=f(x, y)$, where $L$ is a linear differential operator in $\partial_{x}$ and $\partial_{y}$ depending on $k \in \mathbb{C}$, where $(x, y) \in \mathbb{R}^{2}$. The spectral analysis of this equation consists of the construction of two maps: In the construction of the direct map, the equation $L \mu=f$ is solved for $\mu$ in terms of $f$ for all complex $k$. In the construction of the inverse map, $\mu$ is expressed in terms of an appropriate spectral function of $f$ by formulating either a Riemann-Hilbert problem or a $\bar{\partial}$ problem in the complex $k$-plane.

Suppose that the spectral function of $f$ involves an integral transform of $f$. Then the above formalism provides a method for inverting this integral transform.

We recall that the classical derivation of transform pairs involves the integration in the complex $k$-plane of an appropriate Green's function. However, this derivation is based on the assumption that the Green's function is an analytic function of $k$ and it also assumes completeness. The assumption of analyticity corresponds to the case that $\mu$ is sectionally analytic. Therefore, the approach reviewed here has the advantage that, not only it provides a simpler approach to deriving classical transforms avoiding the problem of completeness, but also it can be applied to problems that the associated Green function is not an analytical function of $k$.

The above general formalism will be illustrated in section 2 for the particular case that the relevant integral transform is the attenuated Radon transform.

Let the scalar valued function $\mu\left(x_{1}, x_{2}, y_{1}, y_{2}, k\right)$ satisfy the eigenvalue equation $L \mu=f\left(x_{1}, x_{2}, y_{1}, y_{2}\right) \mu$, where $L$ is a linear differential operator in $\partial_{x_{j}}$ and $\partial_{y_{j}}, j=1,2$, depending on $k \in \mathbb{C}$ and $\left(x_{1}, x_{2}, y_{1}, y_{2}\right) \in \mathbb{R}^{4}$. Proceeding as above and formulating a nonlocal $\bar{\partial}$-problem (instead of a Riemann-Hilbert or a $\bar{\partial}$ problem), we can derive a nonlinear Fourier transform in four spatial dimensions. Such a transform is presented in section 3 ; this transform can be used for the solution of the Cauchy problem of a $4+2$ generalization of the celebrated Kadomtsev-Petriashvilli equation. Reductions to $3+1$ will also be mentioned.

The main difficulty with boundary value problems stems from the fact that only a subset of the boundary values is prescribed as boundary conditions. The determination of the unknown boundary values in terms of the

\footnotetext{
${ }^{1}$ Department of Applied Mathematics and Theoretical Physics, University of Cambridge, Cambridge, CB3 0WA, UK
}

(c) EDP Sciences, SMAI 2009 
given boundary conditions is often called the Dirichlet to Neumann map. Although the terminology is usually used for elliptic problems, the question of determining the Dirichlet to Neumann map, or more precisely the generalized Dirichlet to Neumann map, is also important for evolution PDEs. For example, let $q(x, t)$ satisfy

$$
\begin{gathered}
i q_{t}+q_{x x}=0, \quad 0<x<\infty, \quad 0<t<T, \\
q(x, 0)=q_{0}(x), \quad 0<x<\infty, \\
q(0, t)=g_{0}(t), \quad 0<t<T,
\end{gathered}
$$

where $q_{0}(x)$ and $g_{0}(t)$ have appropriate smoothness, $q_{0}(x)$ has appropriate decay as $|x| \rightarrow \infty$, and $q_{0}(0)=g_{0}(0)$. In this particular case determining the Dirichlet to Neumann map means expressing $q_{x}(0, t)$ in terms of $q_{0}(x)$ and $g_{0}(t)$. This problem can be solved by the classical Fourier transform inversion formula (after the change of variables $k \rightarrow k^{2}$ ). The analogous problem for the nonlinear Schrodinger equation is discussed in section 3 .

\section{The Radon and Attenuated Radon Transforms}

Let the line $L$ make an angle $\theta$ with the positive $x_{1}$-axis. A point $\left(x_{1}, x_{2}\right)$ on this line can be specified by the variables $(\tau, \rho)$, where $\rho$ is the distance from the origin and $\tau$ is a parameter along the line, see Figure 2.1.

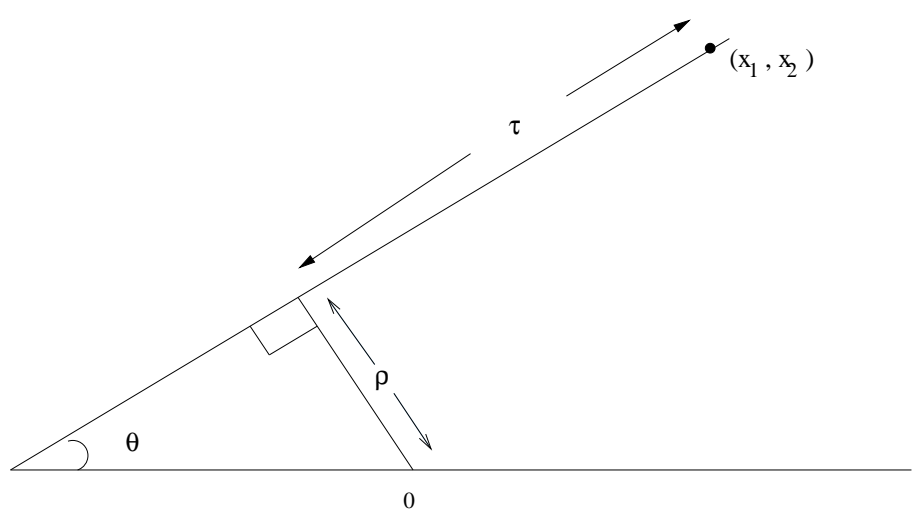

Figure 2.1

A unit vector $\underline{k}$ along $L$ is given by $(\cos \theta, \sin \theta)$, thus

$$
\left(x_{1}, x_{2}\right)=\tau(\cos \theta, \sin \theta)+\rho(-\sin \theta, \cos \theta) .
$$

Hence

$$
\begin{array}{ll}
x_{1}=\tau \cos \theta-\rho \sin \theta, & \rho=x_{2} \cos \theta-x_{1} \sin \theta, \\
x_{2}=\tau \sin \theta+\rho \cos \theta, & \tau=x_{2} \sin \theta+x_{1} \cos \theta .
\end{array}
$$

The Radon transform of a function $f\left(x_{1}, x_{2}\right) \in S\left(\mathbb{R}^{2}\right)$, which will be noted by $\hat{f}(\rho, \theta)$, is defined as the integral of $f$ along $L$, i.e.,

$$
\hat{f}(\rho, \theta)=\int_{-\infty}^{\infty} f(\tau \cos \theta-\rho \sin \theta, \tau \sin \theta+\rho \cos \theta) d \tau, \quad \rho \in \mathbb{R}, \quad 0<\theta<\pi .
$$

The attenuated Radon transform of a function $g\left(x_{1}, x_{2}\right) \in S\left(\mathbb{R}^{2}\right)$ with attenuation $f\left(x_{1}, x_{2}\right) \in S\left(\mathbb{R}^{2}\right)$, which will be denoted by $\hat{g}_{f}(\rho, \theta)$, is defined by

$$
\hat{g}_{f}(\rho, \theta)=\int_{-\infty}^{\infty} e^{-\int_{\tau}^{\infty} f(s \cos \theta-\rho \sin \theta, s \sin \theta+\rho \cos \theta) d s} .
$$




$$
g(\tau \cos \theta-\rho \sin \theta, \tau \sin \theta+\rho \cos \theta) d \tau, \quad \rho \in \mathbb{R}, \quad 0<\theta<\pi
$$

The Radon transform can be inverted in a simple way using the Fourier transform. Also, it can be inverted via the spectral analysis of the following eigenvalue equation for the scalar function $\mu\left(x_{1}, x_{2}, k\right)$ :

$$
\frac{1}{2}\left(k+\frac{1}{k}\right) \frac{\partial \mu}{\partial x_{1}}+\frac{1}{2 i}\left(k-\frac{1}{k}\right) \frac{\partial \mu}{\partial x_{2}}=f\left(x_{1}, x_{2}\right), \quad-\infty<x_{1}, x_{2}<\infty, \quad k \in \mathbb{C} .
$$

Indeed, using this equation it was shown in [1] that

$$
f\left(x_{1}, x_{2}\right)=-\frac{i}{4 \pi^{2}}\left(\partial_{x_{1}}-i \partial_{x_{2}}\right) \int_{0}^{2 \pi} e^{i \theta} J\left(x_{1}, x_{2}, \theta\right) d \theta
$$

where $J$ is the Hilbert transform with respect to $\rho$ of $\hat{f}(\rho, \theta)$ evaluated at $\rho=x_{2} \cos \theta-x_{1} \sin \theta$, i.e.,

$$
J\left(x_{1}, x_{2}, \theta\right)=\int_{-\infty}^{\infty} \frac{\hat{f}\left(\rho^{\prime}, \theta\right) d \rho^{\prime}}{\rho^{\prime}-\left(x_{2} \cos \theta-x_{1} \sin \theta\right)} .
$$

It was shown by Novikov in [2] that the spectral analysis of the eigenvalue equation

$$
\frac{1}{2}\left(k+\frac{1}{k}\right) \frac{\partial \mu}{\partial x_{1}}+\frac{1}{2 i}\left(k-\frac{1}{k}\right) \frac{\partial \mu}{\partial x_{2}}+f\left(x_{1}, x_{2}\right) \mu=g\left(x_{1}, x_{2}\right), \quad-\infty<x_{1}, x_{2}<\infty, \quad k \in \mathbb{C},
$$

yields the following formula for the inverse attenuated Radon transform: $g\left(x_{1}, x_{2}\right)$ is given by the rhs of equation (2.5), where now $J$ is defined by

$$
J\left(x_{1}, x_{2}, \theta\right)=-e^{\int_{\tau}^{\infty} f(s \cos \theta-\rho \sin \theta, s \sin \theta+\rho \cos \theta) d s}\left(e^{P^{-} \hat{f}(\rho, \theta)} P^{-} e^{-P^{-} \hat{f}(\rho, \theta)}+e^{-P^{+} \hat{f}(\rho, \theta)} P^{+} e^{P^{+}} \hat{f}(\rho, \theta)\right) \hat{g} f(\rho, \theta)
$$

in this equation, $\rho$ and $\theta$ are given in terms of $x_{1}$ and $x_{2}$ by the second set of equations $(2.1)$ and $P^{\mp}$ denote the usual projectors in the variable $\rho$, i.e.

$$
\left(P^{\mp} f\right)(\rho)=\mp \frac{f}{2}+\frac{1}{2 i \pi} H f
$$

It was shown in [3] that equation (2.7) can be reduced to equation (2.4), hence the inversion formula for the attenuated Radon transform is the direct consequence of the spectral analysis of the equation (2.4) performed in [1].

In order to construct the direct map associated with equation $(2.4)$ we change variables from $\left(x_{1}, x_{2}\right)$ to $(z, \bar{z})$, where

Then equation (2.4) becomes

$$
z=\frac{1}{2 i}\left(k-\frac{1}{k}\right) x_{1}-\frac{1}{2}\left(k+\frac{1}{k}\right) x_{2}
$$

$$
\nu(k) \frac{\partial \mu}{\partial \bar{z}}=f, \quad \nu(k):=\frac{1}{2 i}\left(\frac{1}{|k|^{2}}-|k|^{2}\right) .
$$

We supplement equation (2.11) with the boundary condition

$$
\mu=O\left(\frac{1}{z}\right), \quad z \rightarrow \infty
$$

Using the inverse $\partial$-bar formula, it follows that the unique solution of the first of equations (2.11) satisfying the boundary condition $(2.12)$, is given by 


$$
\mu\left(x_{1}, x_{2}, k\right)=\frac{1}{2 \pi i} \operatorname{sgn}\left(\frac{1}{|k|^{2}}-|k|^{2}\right) \iint_{\mathbb{R}^{2}} \frac{f\left(x_{1}^{\prime}, x_{2}^{\prime}\right) d x_{1}^{\prime} d x_{2}^{\prime}}{z^{\prime}-z}, \quad|k| \neq 1 .
$$

This equation provides the direct map, i.e., it expresses $\mu$ in terms of $f\left(x_{1}, x_{2}\right)$. In order to construct the inverse problem, i.e. in order to express $\mu$ in terms of an appropriate spectral function, we note that $\mu$ is an analytic function of $k$ in the entire complex $k$-plane (including infinity) except for the unit circle. Thus in order to reconstruct $\mu$, it is sufficient to compute the "jump" $\mu^{+}-\mu^{-}$, where $\mu^{+}$and $\mu^{-}$denote the limits of $\mu$ as $k$ approaches the unit circle from inside and outside the unit disk. A simple computation yields [3]

$$
\mu^{ \pm}=\lim _{\varepsilon \rightarrow 0} \mu\left(x_{1}, x_{2},(1 \mp \varepsilon) e^{i \theta}\right)=\mp P^{\mp} \hat{f}(\rho, \theta)-\int_{\tau}^{\infty} f d s
$$

where $P^{\mp}$ denote the usual projectors in the variable $\rho$ defined in equation $(2.9)$ and $H$ denotes the Hilbert transform.

Equations (2.14) imply

$$
\mu^{+}-\mu^{-}=-\frac{H \hat{f}(\rho, \theta)}{i \pi} .
$$

Substituting this expression in the equation

$$
\mu=\frac{1}{2 i \pi} \int_{0}^{2 \pi} \frac{\left(\mu^{+}-\mu^{-}\right)\left(e^{i \theta^{\prime}}\right) i e^{i \theta^{\prime}} d \theta^{\prime}}{e^{i \theta^{\prime}}-k}, \quad|k| \neq 1,
$$

we find $\mu$ in terms of $\hat{f}$,

$$
\mu\left(x_{1}, x_{2}, k\right)=-\frac{1}{2 i \pi^{2}} \int_{0}^{2 \pi} \frac{e^{i \theta^{\prime}} H \hat{f} d \theta^{\prime}}{e^{i \theta^{\prime}}-k} .
$$

This equation provides the inverse map. Substituting this expression in equation (2.4) we find the inverse Radon transform formula (2.5) and (2.6).

We now present the derivation of the inverse attenuated Radon transform. Equation (2.7) can be rewritten in the form

$$
\nu(k) \frac{\partial \mu}{\partial \bar{z}}+f\left(x_{1}, x_{2}\right) \mu=g\left(x_{1}, x_{2}\right),
$$

where $f \in S\left(\mathbb{R}^{2}\right), g \in S\left(\mathbb{R}^{2}\right), k \in \mathbb{C}$, and $|k| \neq 1$. Equation (2.18) implies

$$
\mu \exp \left[\partial_{\bar{z}}^{-1} \frac{f}{\nu}\right]=\partial_{\bar{z}}^{-1}\left(\frac{g}{\nu} \exp \left[\partial_{\bar{z}}^{-1} \frac{f}{\nu}\right]\right) .
$$

This equation provides the solution of the direct problem, i.e. it expresses $\mu$ in terms of $f$ and $g$. Since $\mu$ is an analytic function of $k$ in the entire complex $k$-plane except for the unit circle, it follows that $\mu$ satisfies the alternative representation (2.16). Thus, in order to express $\mu$ in terms of an appropriate spectral function, all that remains is to compute $\mu^{+}-\mu^{-}$. But this can be easily derived using equation (2.14). Indeed, equation (2.11) can be rewritten in the form

$$
\lim _{k \rightarrow k^{ \pm}}\left\{\partial_{\bar{z}}^{-1}\left(\frac{f\left(x_{1}, x_{2}\right)}{\nu(k)}\right)\right\}=\mp P^{\mp} \hat{f}(\rho, \theta)-\int_{\tau}^{\infty} f d s .
$$

Using this equation, equation (2.19) can be used to compute $\mu^{ \pm}$, and then equation (2.16) provides an alternative representation of $\mu$ in terms of $\hat{g}_{f}(\rho, \theta)$ and of $f$. Substituting this representation in equation (2.7) we find the inverse attenuated Radon transform (2.5) and (2.8). 
Remark 2.1. The Radon transform provides the mathematical foundation of Computerized Tomography as well as of Positron Emission Tomography, whereas the attenuated Radon transform provides the mathematical foundation of Single Photon Emission Computerized Tomography (SPECT) [4]. The latter technique has important applications in many areas of medicine including oncology, cardiology and neurology. The numerical implementation of the inverse attenuated Radon transform using either cubic splines or Chebysev approximations is presented in [3] and [5]. A typical numerical implementation using the numerical technique of filter back projection is shown in the image (c) of Figure 2.2. Figures (b), (c), (d) depict the numerical reconstructions of the realistic cardiac phantom depicted in Figure (a), using three different models. The reconstruction (b) uses the approximation of zero attenuation which reduces the attenuated Radon transform to the classical Radon transform. The reconstruction in (d) uses an improved mathematical model for SPECT (which takes into account the fact that the collimator actually receives "cones" instead of rays); this leads to a modified attenuated Radon transform which can also be inverted analytically [6]. The incorporation of noise to these analytical algorithms is a difficult problem which is under consideration.

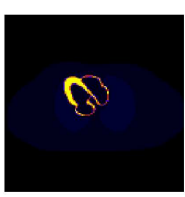

(a)

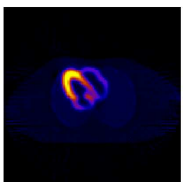

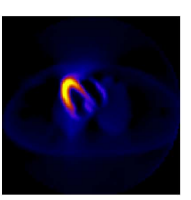

(b)

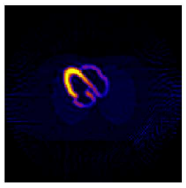

(a)

Figure 2.2: Different reconstructions of a cardiac phantom.

\section{A Nonlinear Fourier Transform in 4 Dimensions and the Generalized KP EQUATION}

Let

$$
x=x_{1}+i x_{2}, y=y_{1}+i y_{2}, k=k_{1}+i k_{2}, \lambda=\lambda_{1}+i \lambda_{2},
$$

where $x_{1}, x_{2}, y_{1}, y_{2}, k_{1}, k_{2}, \lambda_{1}, \lambda_{2}$ are real variables. We will use the shorthand notation $F(x, y, k, \lambda)$ for the function $F\left(x_{1}, x_{2}, y_{1}, y_{2}, k_{1}, k_{2}, \lambda_{1}, \lambda_{2}\right)$.

Let $\mu(x, y, k)$ satisfy the scalar eigenvalue equation

$$
\mu_{\bar{y}}-\mu_{\bar{x} \bar{x}}-2 k \mu_{\bar{x}}+f(x, y) \mu=0 .
$$

By performing the spectral analysis of this equation and by employing a non-local $\bar{\partial}$ formulation the following nonlinear Fourier transform (FT) of $\mathrm{f}$ is constructed in [7]:

Direct nonlinear FT: $\mathbb{R}^{4} \rightarrow \mathbb{R}^{4}: f\left(x_{1}, x_{2}, y_{1}, y_{2}\right) \rightarrow \hat{f}\left(k_{1}, k_{2}, \lambda_{1}, \lambda_{2}\right)$

$$
\hat{f}(k, \lambda)=\frac{2}{\pi^{3}} \int_{\mathbb{R}^{4}}(\bar{\lambda}-\bar{k}) \bar{E}(k, \lambda, x, y) f(x, y) \mu(x, y, \lambda) d x d y,
$$

where $E$ denotes the exponential

$$
E(k, \lambda, x, y)=e^{2 i\left[\left(\lambda_{2}-k_{2}\right) x_{1}+\left(k_{1}-\lambda_{1}\right) x_{2}+2\left(\lambda_{1} \lambda_{2}-k_{1} k_{2}\right) y_{1}+\left(k_{1}^{2}-k_{2}^{2}+\lambda_{2}^{2}-\lambda_{1}^{2}\right) y_{2}\right]},
$$


and $\mu$ is uniquely defined in terms of $f$ by the following integral equation:

$$
\mu(x, y, k)=1+\int_{\mathbb{R}^{4}} G\left(x-x^{\prime}, y-y^{\prime}, k\right) f\left(x^{\prime}, y^{\prime}\right) \mu\left(x^{\prime}, y^{\prime}, k\right) d x^{\prime} d y^{\prime},
$$

where the function $G$ is defined by

$$
G(x, y, k)=-\frac{1}{\pi^{4}} \int_{\mathbb{R}^{4}} \frac{e^{\xi x-\bar{\xi} \bar{x}+\eta y-\bar{\eta} \bar{y}}}{-\bar{\eta}-\bar{\xi}^{2}+2 k \bar{\xi}} d \xi d \eta, \quad \xi=\xi_{1}+i \xi_{2}, \quad \eta=\eta_{1}+i \eta_{2} .
$$

Inverse nonlinear FT: $\mathbb{R}^{4} \rightarrow \mathbb{R}^{4}: \hat{f}\left(k_{1}, k_{2}, \lambda_{1}, \lambda_{2}\right) \rightarrow f\left(x_{1}, x_{2}, y_{1}, y_{2}\right)$

$$
f(x, y)=\frac{2}{\pi} \partial_{\bar{x}} \int_{\mathbb{R}^{4}} E(k, \lambda, x, y) \hat{f}(k, \lambda) \mu(x, y, \lambda) d k d \lambda,
$$

where $\mu$ is uniquely defined in terms of $\hat{f}$ by the linear integral equation

$$
\mu(x, y, k)=1+\frac{1}{\pi} \int_{\mathbb{R}^{4}} E\left(k^{\prime}, \lambda, x, y\right) \hat{f}\left(k^{\prime}, \lambda\right) \mu(x, y, \lambda) \frac{d k^{\prime} d \lambda}{k-k^{\prime}}, \quad k \in \mathbb{C} .
$$

If appropriate norms of $f$ and $\hat{f}$ are sufficiently small then $\mu$ can be approximated by 1 and equations (3.1) reduce to the classical FT pair in four dimensions [7].

It was shown in [7] that the nolinear FT pair (3.1) can be used to solve the Cauchy problem for the following equation in the variable $q(x, y, t)$ :

$$
\begin{gathered}
q_{\bar{t}}=\frac{1}{4} q_{\bar{x} \bar{x} \bar{x}}-\frac{3}{2} q q_{\bar{x}}+\frac{3}{4} \partial_{\bar{x}}^{-1} q_{\bar{y} \bar{y}}, \quad\left(\partial_{\bar{x}}^{-1} f\right)(x)=\frac{1}{\pi} \iint_{\mathbb{R}^{2}} \frac{f(x) d x_{1}^{\prime} d x_{2}^{\prime}}{x-x^{\prime}}, \\
t=t_{1}+i t_{2},\left(t_{1}, t_{2}\right) \in \mathbb{R}^{2}, q(x, y, 0)=q_{0}(x, y) .
\end{gathered}
$$

Equation (3.3) is an integrable generalisation of the KP equation in $4+2$ dimensions, i.e. four spatial $\left(x_{1}, x_{2}, y_{1}, y_{2}\right)$ and two temporal $\left(t_{1}, t_{2}\right)$ dimensions.

The following reductions of the potential version of equation (3.3) are also integrable [8]:

where

$$
\begin{gathered}
q_{t_{1}}=\frac{1}{4} q_{x_{1} x_{1} x_{1}}-\frac{3}{8}\left(q_{x_{1}}^{2}-q_{x_{2}}^{2}\right)+\frac{3}{4} \partial_{x_{1}}^{-1} q_{y_{1} y_{1}}, \quad \Delta q=0, \\
q_{t_{2}}=-\frac{1}{4} q_{x_{2} x_{2} x_{2}}-\frac{3}{4} q_{x_{1}} q_{x_{2}}-\frac{3}{4} \partial_{x_{2}}^{-1} q_{y_{1} y_{1}}, \quad \Delta q=0,
\end{gathered}
$$

$$
\Delta=\partial_{x_{1}}^{2}+\partial_{x_{2}}^{2} .
$$

\section{The Dirichlet to Neumann Map for the NlS}

Equation (1.1) is equivalent to the statement that the following differential form is closed:

$$
W=e^{i k x+i k^{2} t}\left[q d x+\left(i q_{x}+k q\right) d t\right], \quad 0<x<\infty, 0<t<T, k \in \mathbb{C} .
$$

Assuming that $q(x, t)$ has sufficient decay as $x \rightarrow \infty$ for all $0<t<\infty$, and employing Green's theorem, equation (4.1) implies the following global relation:

$$
\hat{q}_{0}(k)-i \int_{0}^{T} e^{i k^{2} s} q_{x}(0, s) d s+k \int_{0}^{T} e^{i k^{2} s} q(0, s) d s=e^{i k^{2} T} \hat{q}_{T}(k), \quad \operatorname{Im} k \geq 0,
$$


where $\hat{q}_{0}(k)$ and $\hat{q}_{T}(k)$ denote the Fourier transform of $q_{0}(x)$ and $q(x, T)$. The first and the third terms of the lhs of equation (4.2) are known, but the term $\hat{q}_{T}(k)$ involves the unknown function $q(x, T)$. Actually, causality implies that $q_{x}(0, t)$ cannot depend on the "future time" $T$, hence the term $\hat{q}_{T}(k)$ cannot contribute to $q_{x}(0, t)$. This motivates the following approach for solving equation (4.2) for $q_{x}(0, t)$ : The classical Fourier transform inversion formula (after the change of variables $k^{2} \rightarrow l$ ) indicates that in order to invert the integral appearing in the lhs of equation (4.2) we must multiply this integral with $k \exp \left[-i k^{2} t\right]$. The function $\hat{q}_{T}(k)$ is analytic for $\operatorname{Im} k>0$ and the function $\exp \left[i k^{2}(T-t)\right]$ is bounded and analytic for $k$ in the union of the first and third quadrants of the complex $k$-plane. Hence, the product $k \exp \left[i k^{2}(T-t)\right] \hat{q}_{T}(k)$ is bounded and analytic in the first quadrant of the complex $k$-plane and is of $O(1)$ as $k \rightarrow \infty$. Thus, by integrating around the boundary of the first quadrant denoted by $\partial I$ and by appealing to Jordan's lemma (after the change of variables $k^{2} \rightarrow l$ ), it follows that the integral of the above product vanishes. Hence, equation (4.2) yields the following expression for $g_{1}(t)=q_{x}(0, t)$ :

$$
g_{1}(t)=\int_{\partial I} k e^{-i k^{2} t}\left[\int_{0}^{\infty} e^{i k x} q_{0}(x) d x-k \int_{0}^{T} e^{i k^{2} s} g_{0}(s) d s\right] d k, \quad 0<t<T .
$$

By changing the order of the $x$ - and $s$-integrations with the $k$-integration and then by computing the $k$-integrals (see [9] for details), we find that equation (4.3) simplifies to the following equation

$$
g_{1}(t)=-\frac{1}{\sqrt{\pi}} e^{-\frac{i \pi}{4}}\left[\frac{1}{\sqrt{t}} \int_{0}^{\infty} e^{\frac{i x^{2}}{4 t}} \dot{q}_{0}(x) d x+\int_{0}^{t} \frac{\dot{g}_{0}(s)}{\sqrt{t-s}} d s\right], \quad 0<t<T .
$$

The occurrence of the derivatives $\dot{q}_{0}$ and $\dot{g}_{0}$ is due to the fact that in order to obtain well defined $k$-integrals we first integrate by parts the $x$ - and $s$-integrals before changing the order of integration.

Let $q(x, t)$ satisfy the following initial-boundary value problem:

$$
\begin{gathered}
i q_{t}+q_{x x}-2|q|^{2} q=0, \quad 0<x<\infty, 0<t<T \\
q(x, 0)=0, \quad 0<x<\infty \\
q(0, t)=g_{0}(t), \quad 0<t<T
\end{gathered}
$$

where $g_{0}(t)$ has sufficient smoothness and $g_{0}(0)=0$. Our goal is to determine $g_{1}(t)=q_{x}(0, t)$ in terms of $g_{0}(t)$. In this case the analogue of the global relation (4.1) is the following equation [9]

$$
\int_{0}^{T} e^{2 i k^{2} \tau}\left\{i\left|g_{0}(\tau)\right|^{2} \Phi_{1}(\tau, k)-\left[2 k g_{0}(\tau)+i g_{1}(\tau)\right] \Phi_{2}(\tau, k)\right\} d \tau=e^{4 i k^{2} T} c^{+}(k), \quad \operatorname{Im} k \geq 0
$$

where $c^{+}(k)$ is an analytic function of $k$ for $\operatorname{Im} k>0$ and of order $O(1 / k)$ as $k \rightarrow \infty$. The functions $\Phi_{1}$ and $\Phi_{2}$ are certain solutions of the $t$-dependant part of the associated Lax pair. The integral involving $g_{1}(\tau)$, in addition to the exponential $\exp \left[2 i k^{2} \tau\right]$, it also contains the function $\Phi_{2}(\tau, k)$.

This function makes the $k$-dependence prohibitively complicated for the application of the Fourier inversion formula. This observation motivates the following question. Does there exist a representation of $\left(\Phi_{1}, \Phi_{2}\right)$ involving exponential dependence on $k$ ? The answer is positive and such a formula is provided by the so called Gelfand-Levitan-Marchenko (GLM) representation [10]: The vector $\Phi$ can be represented in the form

$$
\Phi(t, k)=\left(\begin{array}{c}
0 \\
e^{i f_{2} t}
\end{array}\right)+\int_{-t}^{t}\left(\begin{array}{c}
L_{1}(t, s)-\frac{i}{2} g_{0}(t) M_{2}(t, s)+k M_{1}(t, s) \\
L_{2}(t, s)+\frac{i \rho}{2} \bar{g}_{0}(t) M_{1}(t, s)+k M_{2}(t, s)
\end{array}\right) e^{i f_{2} s} d s
$$

where the functions $\left\{L_{j}, M_{j}, N_{j}\right\}_{1}^{2}$ satisfy the following equations:

$$
L_{1}(t, t)=\frac{i}{2} g_{1}(t), \quad M_{1}(t, t)=g_{0}(t), \quad L_{2}(t,-t)=M_{2}(t,-t)=0
$$




$$
\begin{aligned}
& L_{1_{t}}-L_{1_{s}}=i g_{1}(t) L_{2}+\alpha(t) M_{1}+\beta(t) M_{2}, \\
& L_{2_{t}}+L_{2_{s}}=-i \rho \bar{g}_{1}(t) L_{1}-\alpha(t) M_{2}+\rho \bar{\beta}(t) M_{1}, \\
& M_{1_{t}}-M_{1_{s}}=2 g_{0}(t) L_{2}+i g_{1}(t) M_{2}, \\
& M_{2_{t}}+M_{2_{s}}=2 \rho \bar{g}_{0}(t) L_{1}-i \rho \bar{g}_{1}(t) M_{1},
\end{aligned}
$$

with $\alpha(t)$ and $\beta(t)$ defined by the equations

$$
\alpha(t)=\frac{\rho}{2}\left(g_{0} \bar{g}_{1}-\bar{g}_{0} g_{1}\right), \quad \beta(t)=\frac{1}{2}\left(i \dot{g}_{0}-\rho\left|g_{0}\right|^{2} g_{0}\right) .
$$

Using the GLM representation we will now show that the Neumann boundary values $g_{1}(t)=q_{x}(0, t)$ can be expressed in terms of the Dirichlet boundary value $g_{0}(t)$ by the following formula [11],[12]:

$$
g_{1}(t)=g_{0}(t) M_{2}(t, t)-\frac{e^{-\frac{i \pi}{4}}}{\sqrt{\pi}} \int_{0}^{t} \frac{\partial M_{1}}{\partial \tau}(t, 2 \tau-t) \frac{d \tau}{\sqrt{t-\tau}} .
$$

Indeed, replacing the function $\Phi_{1}$ by the rhs of (4.7) in the global relation (4.6) we find

$$
\begin{gathered}
\int_{0}^{t} e^{4 i k^{2} \tau}\left[L_{1}(t, 2 \tau-t)-\frac{i}{2} g_{0}(t) M_{2}(t, 2 \tau-t)+k M_{1}(t, 2 \tau-t)\right] d \tau \\
=-\frac{e^{4 i k^{2} t}}{2} C(k, t), \quad \operatorname{Im} k \geq 0, \quad 0<t<T .
\end{gathered}
$$

We multiply this equation by $k \exp \left[-4 i k^{2} t^{\prime}\right], t^{\prime}<t$, and integrate along the boundary of the first quadrant of the complex $k$-plane, which we denote by $\partial I$, with the orientation shown in Figure 4.1.

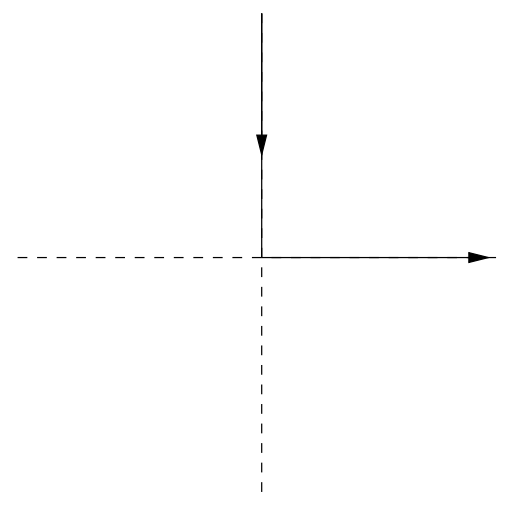

Figure 4.1: The curve $\partial I$.

The rhs of the resulting equation vanishes because $k c^{+}(k, t)$ is analytic and of $O(1)$ for $\operatorname{Im} k>0$, and the oscillatory term $\exp \left[i k^{2}\left(t-t^{\prime}\right)\right]$ is bounded in the first quadrant.

The first two terms of the lhs of equation (4.12) give contributions which can be computed in closed form using the following identity

$$
\int_{\partial I} k\left[\int_{0}^{t} e^{4 i k^{2}\left(\tau-t^{\prime}\right)} K(\tau, t) d \tau\right] d k=\frac{\pi}{4} K\left(t^{\prime}, t\right), \quad t>0, \quad t^{\prime}>0, \quad t^{\prime}<t
$$

where $K(\tau, t)$ is a smooth function of its arguments. This identity follows from the usual Fourier transform identity after using the transformation $4 k^{2} \rightarrow l$ to map $\partial I$ to the real axis. Using this identity, the first two 
terms of the lhs of equation (4.12) yield

$$
\frac{\pi}{4}\left[L_{1}\left(t, 2 t^{\prime}-t\right)-\frac{i}{2} g_{0}(t) M_{2}\left(t, 2 t^{\prime}-t\right)\right] .
$$

Before computing the contribution of the third term in the lhs of equation (4.12), we first use integration by parts:

$$
\begin{gathered}
\int_{0}^{t} k e^{4 i k^{2} \tau} M_{1}(t, 2 \tau-t) d \tau=\frac{1}{4 i k}\left[e^{4 i k^{2} t} M_{1}(t, t)-M_{1}(t,-t)\right] \\
-\frac{1}{4 i k} \int_{0}^{t} e^{4 i k^{2} \tau} \frac{\partial M_{1}}{\partial \tau}(t, 2 \tau-t) d \tau .
\end{gathered}
$$

Multiplying this term by $k \exp \left[-4 i k^{2} t^{\prime}\right]$ and integrating along $\partial I$, we find three contributions. The first vanishes due to the fact that $\exp \left[4 i k^{2}\left(t-t^{\prime}\right)\right]$ is bounded in the first quadrant of the complex $k$-plane. The $k$-integral of the second contribution can be computed in closed form: using $k^{2} t^{\prime}=l^{2}$ we find

$$
\int_{\partial I} e^{-4 i k^{2} t^{\prime}} d k=\int_{\partial I} e^{-4 i l^{2}} \frac{d l}{\sqrt{t^{\prime}}}=\frac{c}{\sqrt{t^{\prime}}}
$$

with

$$
c=\int_{\partial I} e^{-4 i l^{2}} d l=\frac{1}{2} \int_{\partial I} e^{-i l^{2}} d l=\frac{1}{2} \int_{-\infty}^{\infty} e^{-i l^{2}} d l=\int_{0}^{\infty} e^{-i l^{2}} d l=\frac{1}{2} e^{-\frac{i \pi}{4}} \Gamma\left(\frac{1}{2}\right),
$$

where in deforming $\partial I$ to the real axis we have used the fact that $\exp \left(-i l^{2}\right)$ is bounded in the second quadrant of the complex $k$-plane.

In order to compute the contribution of the third term in the rhs of equation (4.15) we split $\int_{0}^{t}$ into $\int_{0}^{t^{\prime}}$ and $\int_{t^{\prime}}^{t}$. The contribution of the second integral vanishes due to analyticity considerations and the contribution of the first integral yields a $k$-integral which equals $c / \sqrt{t^{\prime}-t}$. Thus, equation (4.12) yields

$$
\begin{gathered}
\frac{\pi}{4}\left[L_{1}\left(t, 2 t^{\prime}-t\right)-\frac{i}{2} g_{0}(t) M_{2}\left(t, 2 t^{\prime}-t\right)\right] \\
-\frac{c}{4 i}\left[\frac{M_{1}(t,-t)}{\sqrt{t^{\prime}}}+\int_{0}^{t^{\prime}} \frac{\partial M_{1}}{\partial \tau}(t, 2 \tau-t) \frac{d \tau}{\sqrt{t^{\prime}-\tau}}\right]=0 .
\end{gathered}
$$

Letting $t^{\prime} \rightarrow t$, the above equation becomes equation (4.11).

\section{REFERENCES}

(1) A.S. Fokas and R. G. Novikov, Discrete Analogues of the Dbar Equation and of Radon Transform, C.R. Acad. Sci., Paris 313,75-80 (1991).

(2) R. G. Novikov, An Inversion Formula for the Attenuated X-ray Trasnformation, Ark. Mat. 40, 145 (2002).

(3) A.S. Fokas, A. Iserles and V. Marinakis, Reconstruction Algorithm for Single Photon Emission Computed Tomography and its Numerical Implementation, J. R. Soc. Interface 3, 45 (2006).

(4) M.N. Wernick and J.N. Aarsvold, Eds., Emission Tomography, The Fundamentals of PET and SPECT, Elsevier Academic Press, USA (2004).

(5) A.S. Fokas and V. Marinakis, Reconstruction Algorithm for the Brain Imaging Techniques of PET and SPECT, Hermis Intern. Journal 4, 45-61 (2004).

(6) A.S. Fokas, B. Hutton and K. Kacperski (in preparation). 
(7) A.S. Fokas, The D-Bar Method, Inversion of Certain Integrals and Integrability in $4+2$ and $3+1$ Dimensions, J. Phys. A, 41, 344006 (2008).

(8) A.S. Fokas, Soliton Multidimensional Equations and Integrable Evolutions Preserving Laplace's Equation, Phys. Lett. A 372, 1277-1279 (2008).

(9) A.S. Fokas, A Unified Approach to Boundary Value Problems, CBMS-NSF Regional Conference Series in Applied Mathematics 78, SIAM, USA (2008).

(10) A. Boutet de Monvel and V. Kotlyarov, Scattering Problem for the Zakharov-Shabat Equations on the Semi-Axis, Inverse Problems 16, 1813-1837 (2000).

(11) A. Boutet de Monvel, A.S. Fokas and D. Shepelsky, The Analysis of the Global Relation for the Nonlinear Schrödinger Equation on the Half-Line, Lett. Math. Phys. 65, 199-212 (2003).

(12) A.S. Fokas, A Generalised Dirichlet to Neumann Map for Certain Nonlinear Evolution PDEs, Comm. Pure Appl. Math. LVIII, 639-670 (2005). 